\title{
Introduction
}

\section{Intracerebral hemorrhage: a changing landscape}

David W. Newell, M.D.

\footnotetext{
Swedish Neuroscience Institute, Seattle, Washington
}

This issue of Neurosurgical Focus contains a collection of articles related to changing treatment paradigms for intracerebral hemorrhage. Intracerebral hemorrhage from a variety of causes has been an understudied phenomenon given its prevalence and devastating consequences. New information about natural history, factors influencing outcome, elucidation of additional pathophysiological mechanisms, and neurointensive care management has led to a renewed enthusiasm to improve outcome based on rational treatment paradigms. More recently the introduction of many new anticoagulants and antiplatelet agents has presented new management dilemmas for physicians caring for patients with intracerebral hemorrhage. This collection of interesting papers will hopefully update the reader on current management controversies as well as emerging treatments that may offer promise to improve outcomes in these patients in the future.

The first article by Kelly et al. outlines the challenge of surgical decision making when confronted with patients with intracerebral hemorrhage. This timely review summarizes a number of studies regarding the natural history of the condition and factors that influence outcome and are related to the treatment recommendations made to patients and families. The article points out that factors, including geography, treatment biases, patient perceptions, and physician biases, can have a major influence on decision making and selection of different treatments for patients with intracerebral hemorrhages.

Please include this information when citing this paper: DOI: 10.3171/2013.3.FOCUS13125.
The next article by Goren and associates focuses on modern intraoperative imaging modalities and how they may be employed in identifying vascular lesions that may be the cause of intracerebral hemorrhage. In the past, the etiology of hemorrhages were often presumed based on characteristic CT appearance, but the important recognition of underlying vascular lesions has been made easier by advances in modern imaging modalities that this article nicely outlines.

The next 3 articles deal with the role of decompressive hemicraniectomy in intracerebral hemorrhage patients who are surgically treated and rationales for use of this technique to try to preserve viable brain tissue when treating hemorrhages surgically.

The subsequent articles, one by James et al. and the other by Awad et al., detail the use of anticoagulants, antiplatelet agents, and newer agents including Dabigatran and the need for reversal of the effects of these agents in the management of intracerebral hemorrhage. The common use of some of the newer agents for anticoagulation therapy and antiplatelet therapy and their different durations of action, as well as different strategies of reversal of these agents, has required new protocols to be put in place. Close cooperation with blood banks can help effect the reversal of these agents in these patients to limit the expansion of the hemorrhage and can be a prerequisite for surgery, when contemplating surgical treatment. The article by James et al., "The role of anticoagulants, antiplatelet agents, and their reversal strategies in the management of intracerebral hemorrhage," provides a detailed review of the pathophysiology of these agents and effective treatment strategies. There is a very useful table at the end that will be valuable as a reference guide for clinicians treating these patients.

Neugebauer et al. provide a thorough review of the role of managing cerebellar infarction associated with mass effect and various management strategies including ventricular drainage, observation, and surgery. There is a 
valuable table provided at the end with a very nice literature review that attempts to survey the current literature on published outcomes with different management strategies of this condition.

The next 2 articles, one by Belur et al. and the other by Sussman et al., outline experimental therapies that have promise to limit the secondary effects of physiological mechanisms that are activated after brain hemorrhage, which can lead to delayed deterioration in patients.

The next paper by Gross and colleagues outlines the incidence of and risk factors for hydrocephalus formation after arteriovenous malformation (AVM) rupture. This carefully conducted retrospective review indicates that hydrocephalus following AVM rupture was associated with initial Glasgow Coma Scale score, intraventricular hemorrhage, and AVM-associated aneurysms.

In the subsequent article, Wright and coworkers provide the result of a retrospective case-control study that observes the infection rate in groups of patients in whom ventricular drains were placed, with and without antibiotic impregnation.
The next article, by Monteith et al., describes a novel proposed minimally invasive treatment for intracerebral hemorrhage in which MRI-guided focused ultrasound is used to liquefy intraparenchymal hemorrhages and aspirate them to reduce mass effect. Studies have been done in cadavers that indicate the feasibility of this approach in humans.

The last article, by Daniels et al., describes the clinical features and outcome of patients with intracranial dural arteriovenous fistulas presenting with hemorrhage. The most common location for fistulas presenting with hemorrhage is tentorial. Excellent outcomes can be achieved with individualized multimodality treatment. Despite the hemorrhagic presentation, almost two-thirds of patients experience a full recovery with no or minimal residual symptoms.

(http://thejns.org/doi/abs/10.3171/2013.3.FOCUS13125)

\section{Disclosure}

The author reports no conflict of interest. 